\title{
A Chemoenzymatic Approach toward the Rapid and Sensitive Detection of O-GICNAc Posttranslational Modifications
}

\author{
Nelly Khidekel, ${ }^{\dagger}$ Sabine Arndt, ${ }^{\dagger}$ Nathan Lamarre-Vincent, ${ }^{\dagger}$ Alexander Lippert, ${ }^{\dagger}$ \\ Katherine G. Poulin-Kerstien, ${ }^{\dagger}$ Boopathy Ramakrishnan, ${ }^{\ddagger}$, Pradman K. Qasba, ${ }^{\ddagger}$ and \\ Linda C. Hsieh-Wilson*, $†$ \\ Division of Chemistry and Chemical Engineering, California Institute of Technology, Pasadena, California 91125, \\ and Structural Glycobiology Section, Laboratory of Experimental and Computational Biology, CCR, NCI, \\ SAIC-Frederic, Inc., Frederick, Maryland 21702-1201
}

Received September 16, 2003; E-mail: Ihw@caltech.edu

Protein glycosylation is one of the most abundant posttranslational modifications and plays a fundamental role in the control of biological systems. For example, carbohydrate modifications are important for host-pathogen interactions, inflammation, development, and malignancy. ${ }^{1}$ As part of a broader program to understand the role of protein glycosylation in neuronal communication, we are investigating $O$-GlcNAc glycosylation, which is the covalent modification of serine and threonine residues by $\beta$ - $N$-acetylglucosamine. ${ }^{2}$ The $O$-GlcNAc modification is found in all higher eukaryotic organisms from $C$. elegans to man and has been shown to be ubiquitous, inducible, and highly dynamic, suggesting a regulatory role analogous to phosphorylation. However, the regulatory nature of the modification (i.e., dynamic, low cellular abundance) also represents a central challenge in its detection and study.

A common method to observe $O$-GlcNAc involves labeling proteins with $\beta$-1,4-galactosyltransferase (GalT), an enzyme that catalyzes the transfer of $\left[{ }^{3} \mathrm{H}\right]$ galactose from UDP- $\left[{ }^{3} \mathrm{H}\right]$ galactose to terminal GlcNAc groups. ${ }^{3}$ Unfortunately, this approach is expensive, involves handling of radioactive material, and requires exposure times of days to months. Antibodies ${ }^{4,5}$ and lectins ${ }^{3}$ offer alternative means of detection, but they can suffer from weak binding affinity and limited specificity.

In this Communication, we report a new strategy for the rapid and sensitive detection of $O$-GlcNAc glycosylated proteins. Our approach capitalizes on the substrate tolerance of GalT, which allows for chemoselective installation of an unnatural ketone functionality to $O$-GlcNAc modified proteins (Figure 1). The ketone moiety has been well-characterized in cellular systems as a neutral, yet versatile, chemical handle. ${ }^{6}$ Here, it serves as a unique marker to "tag" $O$-GlcNAc glycosylated proteins with biotin. Once biotinylated, the glycoconjugates can be readily detected by chemiluminescence using streptavidin conjugated to horseradish peroxidase (HRP).

We designed UDP analogue 1 on the basis of previous biochemical and structural studies of GalT (Figure 1). We chose to append the ketone functionality at the $\mathrm{C}-2$ position of the galactose ring because GalT has been shown to tolerate unnatural substrates containing minor substitutions at the C-2 position, including 2-deoxy, 2-amino, and 2- $\mathrm{N}$-acetyl substituents. ${ }^{7}$ Moreover, 2deoxy-Gal was transferred at rates comparable to Gal, whereas 3-, 4-, and 6-deoxy-Gal were transferred at reduced rates. ${ }^{7,8}$ Analysis of the crystal structures of GalT complexed with UDP-GalNAc revealed that the $\mathrm{C}-2 \mathrm{~N}$-acetyl moiety is accommodated in a shallow pocket within the active site. ${ }^{9}$ Importantly, the single Y289L mu-

$\dagger$ California Institute of Technology.

\$ SAIC-Frederic, Inc.

$\S$ CCR, NCI, Frederick, MD.

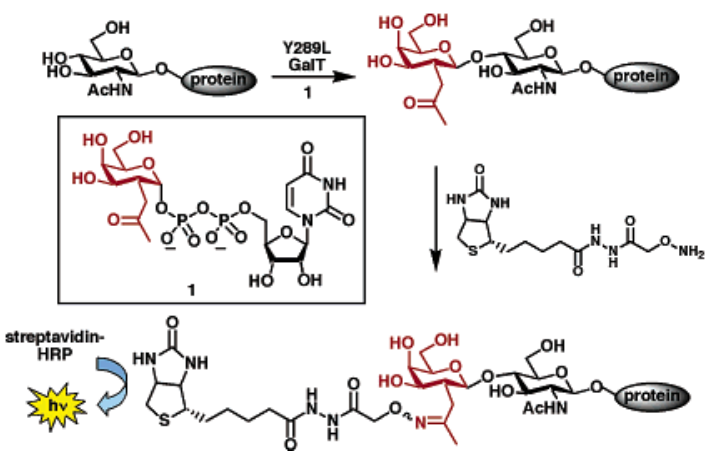

Figure 1. Strategy for the detection of $O$-GlcNAc glycosylated proteins.

Scheme $1^{a}$
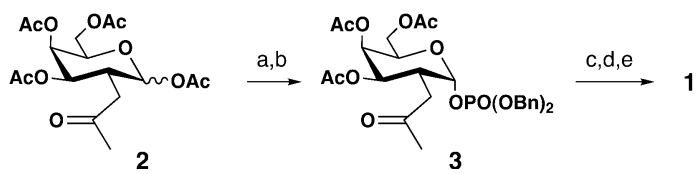

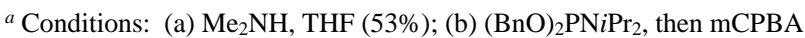
(54\%); (c) $\mathrm{Pd} / \mathrm{C}, \mathrm{H}_{2}$, tri- $n$-octylamine; (d) UMP-morpholidate, $1 H$-tetrazole, pyr; (e) TEA, $\mathrm{H}_{2} \mathrm{O} / \mathrm{MeOH}$ (45\%, three steps).

tation enlarges the binding pocket and enhances the catalytic activity toward GalNAc substrates without compromising specificity. ${ }^{9}$

Analogue 1 was synthesized from the previously reported ketone $2^{10}$ as shown in Scheme 1. Selective anomeric deacetylation followed by treatment with $(\mathrm{BnO})_{2} \mathrm{PNi} \mathrm{Pr}_{2}{ }^{11}$ afforded the phosphite, which was directly oxidized with $\mathrm{mCPBA}^{12}$ to produce dibenzyl phosphate 3 . Hydrogenolytic debenzylation yielded the unprotected phosphate as the trioctylammonium salt, which was coupled with UMP-morpholidate in pyridine ${ }^{13}$ to provide the target molecule $\mathbf{1}$ upon deacetylation with TEA.

With analogue $\mathbf{1}$ in hand, we examined the ability of GalT to label the peptide TAPTS $(O$-GlcNAc)TIAPG, which encompasses an $O$-GlcNAc modification site within the protein CREB. ${ }^{14}$ Using wild-type GalT, only partial transfer of the keto-sugar was observed by LC-MS $(\sim 1.5 \%)$. As anticipated, however, the Y289L mutant showed greater activity and afforded complete conversion after 6 $\mathrm{h}$ at $4{ }^{\circ} \mathrm{C}$ (Figure 2). Subsequent reaction of the ketone-labeled peptide with the aminooxy biotin derivative, $N$-(aminooxyacetyl)$N^{\prime}$-(D-biotinoyl) hydrazine, under mild conditions (pH 6.7 buffer, $8 \mathrm{~h}, 25^{\circ} \mathrm{C}$ ) gave complete formation of the corresponding $O$-alkyl oxime.

Having demonstrated the labeling of a peptide, we applied our strategy to the $O$-GlcNAc glycosylated protein CREB. Recombinant CREB from Sf9 cells ${ }^{14}$ was incubated with 1 and Y289L GalT for $12 \mathrm{~h}$ at $4{ }^{\circ} \mathrm{C}$. Following reaction with aminooxy biotin, the mixture 


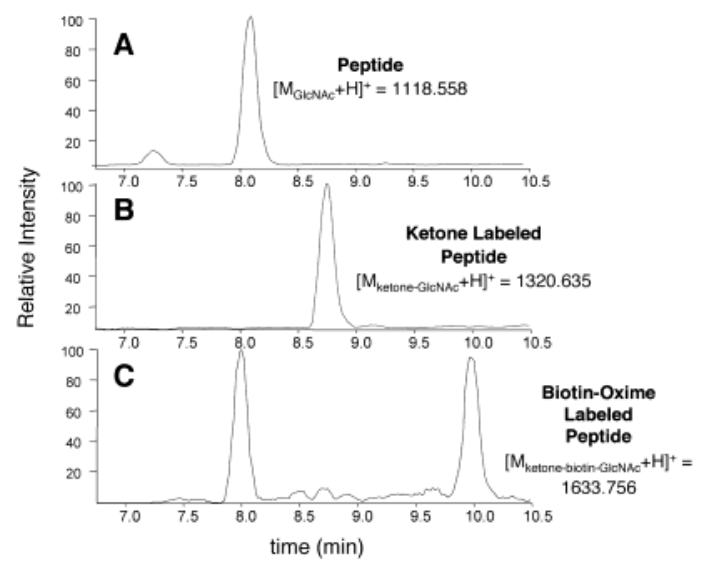

Figure 2. Labeling of the peptide TAPTS $(O$-GlcNAc)TIAPG. LC-MS traces monitoring the reaction progress at (A) time 0 , (B) $6 \mathrm{~h}$ after the addition of $\mathbf{1}$ and Y289L GalT, and (C) $8 \mathrm{~h}$ after biotin addition. A and B represent base peak chromatograms, and $\mathrm{C}$ is the extracted ion chromatogram within $1319.0-1321.0$ and $1633.0-1635.5 \mathrm{~m} / \mathrm{z}$. The peak at $8 \mathrm{~min}$ in $\mathrm{C}$ is a biotin impurity. See Supporting Information for details.

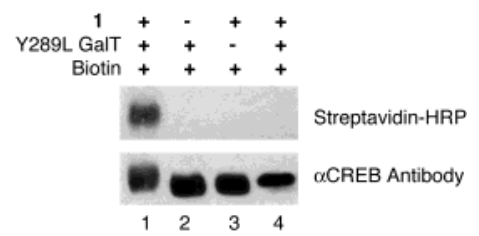

Figure 3. Selective labeling of glycosylated CREB. CREB from Sf 9 cells (lanes 1-3) or E. coli (lane 4) was tested.

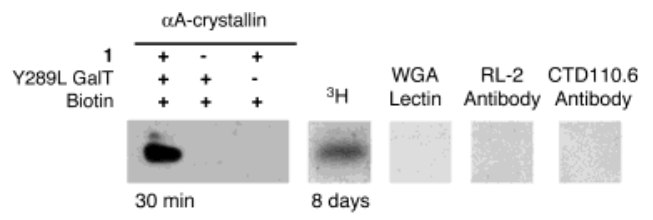

Figure 4. Labeling of $\alpha$-crystallin, and comparison with several existing detection methods. For the ketone and tritium labeling studies, $0.75 \mu \mathrm{g}$ of protein was used; for the lectin and antibodies, $5 \mu \mathrm{g}$ of protein was used. See Supporting Information for conditions and full experimental controls.

was resolved by SDS-PAGE, transferred to nitrocellulose, and probed with streptavidin-HRP. Strong labeling of CREB was observed by chemiluminescence within seconds of exposure to film (Figure 3). In contrast, no signal was observed over the same time period for unglycosylated CREB (from E. coli) or when reactions were performed in the absence of either 1 or enzyme, demonstrating the selectivity of the transfer.

We next explored the sensitivity of the approach using the challenging target, $\alpha$-crystallin. Detection of the $O$-GlcNAc moiety on $\alpha$-crystallin has been reported to be particularly difficult due to its low stoichiometry of glycosylation $(\sim 10 \%)$ and the presence of only one major modification site. ${ }^{15}$ Indeed, we found that existing methods such as wheat-germ agglutinin (WGA) lectin ${ }^{3}$ and the $O$-GlcNAc-specific antibodies RL- $2^{4}$ and CTD110. $6^{5}$ failed to detect any $O$-GlcNAc modification on $\alpha$-crystallin, even when $10 \mu \mathrm{g}$ of $\alpha$-crystallin was used (Figure 4 and Supporting Information). In contrast, our approach enabled detection of the $O$-GlcNAc modification within minutes using $0.75 \mu \mathrm{g}$ of $\alpha$-crystallin. For comparison, tritium labeling with wild-type GalT required 8 days of exposure to film for a weaker signal. Thus, our approach represents at least a 380-fold enhancement in signal over traditional methods.

Recently, Vocadlo et al. have reported the extension of their Staudinger methodology to $O$-GlcNAc glycosylated proteins. ${ }^{16}$ The strategy described here complements the metabolic labeling ap- proach and is distinct in several key respects. First, the use of an engineered GalT and $\mathbf{1}$ enables near stoichiometric labeling, resulting in higher sensitivity. Enhanced sensitivity is crucial in studying $O$-GlcNAc as the regulatory nature of the modification means that it is often present only in low cellular abundance. Second, the use of an engineered GalT rather than the native $O$-GlcNAc glycosyltransferase allows one to capture the glycosylated species directly and avoid perturbation of metabolic pathways. Thus, our approach should permit the observation of $O$-GlcNAc signaling pathways after cellular stimulation, an important frontier in the field.

In conclusion, we have developed a novel chemoenzymatic strategy that detects $O$-GlcNAc modifications with an efficiency and sensitivity that is unrivaled by existing methods. Given the chemical versatility of the ketone handle, we can envision a variety of applications, including direct fluorescence detection, affinity enrichment, and isotopic labeling for comparative proteomics. Moreover, the study of other enzymes (e.g., farnesyltransferases and other glycosyltransferases) may also benefit from this approach. Current efforts in our laboratory are focused on the extension of the approach to novel glycosylated proteins and to the dynamic regulation of the modification in cells.

Acknowledgment. We thank Dr. M. Shahgholi, Dr. P. Snow, H.-C. Tai, and S. Tully for helpful discussions and assistance. This research was supported by an NSF CAREER Award (CHE0239861) and an Alfred P. Sloan Fellowship.

Supporting Information Available: Synthetic procedures, MS analysis, and detailed labeling procedures (PDF). This material is available free of charge via the Internet at http://pubs.acs.org.

\section{References}

(1) (a) Varki, A. Glycobiology 1993, 3, 97-130. (b) Lasky, L. A. Annu. Rev Biochem. 1995, 64, 113-139. (c) Capila, I.; Linhardt, R. J. Angew. Chem. Int. Ed. 2002, 41, 391-412. (d) Rudd, P. M.; Elliott, T.; Cresswell, P.; Wilson, I. A.; Dwek, R. A. Science 2001, 291, 2370-2376.

(2) (a) Wells, L.; Vosseller, K.; Hart, G. W. Science 2001, 291, 2376-2378. (b) Zachara, N. E.; Hart, G. W. Chem. Rev. 2002, 102, 431-438.

(3) Roquemore, E. P.; Chou, T. Y.; Hart, G. W. Methods Enzymol. 1994, 230, 443-460. As GalT labels terminal GlcNAc sugars, experiments using $\mathrm{PNGaseF}$ and $\beta$-elimination serve as valuable controls.

(4) Snow, C. M.; Senior, A.; Gerace, L. J. Cell Biol. 1987, 104, 1143-1156.

(5) Comer, F. I.; Vosseller, K.; Wells, L.; Accavitti, M. A.; Hart, G. W. Anal. Biochem. 2001, 293, 169-177.

(6) (a) Cornish, V. W.; Hahn, K. M.; Schultz, P. G. J. Am. Chem. Soc. 1996 118, 8150-8151. (b) Mahal, L. K.; Yarema, K. J.; Bertozzi, C. R. Science 1997, 276, 1125-1128. (c) Datta, D.; Wang, P.; Carrico, I. S.; Mayo, S L.; Tirrell, D. A. J. Am. Chem. Soc. 2002, 124, 5652-5653.

(7) (a) Qian, X.; Sujino, K.; Palcic, M. M.; Ratcliffe, R. M. In Glycochemistry: Principles, Synthesis, and Application; Wang, P. G., Bertozzi, C. R., Eds.; Marcel Dekker: New York, 2001; pp 535-565. (b) Wong, C. H.; Halcomb, R. L.; Ichikawa, Y.; Kajimoto, T. Angew. Chem., Int. Ed. Engl. 1995, 34, 521-546.

(8) Notably, a biotin moiety appended to the 6-position of UDP-Gal can serve as a substrate for human (but not bovine) GalT: Bütler, T.; Schumacher, T.; Namdjou, D.-J.; Gallego, R. G.; Clausen, H.; Elling, L. ChemBioChem 2001, 2, 884-894.

(9) Ramakrishnan, B.; Qasba, P. K. J. Biol. Chem. 2002, 277, 20833-20839.

(10) Hang, H. C.; Bertozzi, C. R. J. Am. Chem. Soc. 2001, 123, 1242-1243.

(11) Sim, M. M.; Kondo, H.; Wong, C.-H. J. Am. Chem. Soc. 1993, 115, 22602267.

(12) Ha, S.; Chang, E.; Lo, M.-C.; Men, H.; Park, P.; Ge, M.; Walker, S. J. Am. Chem. Soc. 1999, 121, 8415-8426.

(13) Wittmann, V.; Wong, C.-H. J. Org. Chem. 1997, 62, 2144-2147.

(14) Lamarre-Vincent, N.; Hsieh-Wilson, L. C. J. Am. Chem. Soc. 2003, 125, $6612-6613$

(15) (a) Chalkley, R. J.; Burlingame, A. L. J. Am. Soc. Mass Spectrom. 2001 12, 1106-1113. (b) Haynes, P. A.; Aebersold, R. Anal. Chem. 2000, 72, $5402-5410$.

(16) Vocadlo, D. J.; Hang, H. C.; Kim, E. J.; Hanover, J. A.; Bertozzi, C. R. Proc. Natl. Acad. Sci. U.S.A. 2003, 100, 9116-9121.

JA038545R 